\title{
A note on the use of sensitivity analysis to explore the potential impact of declining institutional care utilisation on disability prevalence Patrick J Graham*
}

\author{
Address: Department of Public Health and General Practice, Christchurch School of Medicine and Health Sciences, P.O. Box 4345, Christchurch, \\ New Zealand \\ Email: Patrick J Graham* - patrick.graham@chmeds.ac.nz \\ * Corresponding author
}

Population Health Metrics 2004, 2:3

Accepted: 16 March 2004

This article is available from: http://www.pophealthmetrics.com/content/2/l/3

(C) 2004 Graham; licensee BioMed Central Ltd. This is an Open Access article: verbatim copying and redistribution of this article are permitted in all media for any purpose, provided this notice is preserved along with the article's original URL.

\begin{abstract}
Many health and disability surveys are conducted using the non-institutionalised population as a sampling frame. Consequently, it is possible that changes in the utilisation of institutional care could account for all or part of any change in the observed prevalence of functional limitation, disability or other health state, based on samples from the non-institutionalised population. Using conditional probability arguments, I present an adjustment formula for computing health state prevalences for the non-institutionalised population under a scenario in which health state prevalences are held constant except for movement into the non-institutionalised population of individuals who would formerly have been in institutional care. By comparing the adjusted prevalence with observed noninstitutionalised health state prevalences the contribution of changes in institutionalisation to observed changes in the non-institutionalised health state prevalence can be assessed.
\end{abstract}

\section{Background}

Prevalence measures of disability, functional limitation and other health states are important descriptive statistics for assessing population health and are integral to the construction of summary measures such as health expectancy [1]. Commonly used health and disability survey sampling frames exclude individuals living in institutions such as hospitals or retirement homes. For example, reports based on the United States National Health Interview Survey [2], the French Health and Medical Care Survey [3], the Finnish and Norwegian Surveys of Living Conditions [4] and the New-Zealand Household Health Survey [5] all note that the survey data refer to the noninstitutionalised population. Because the prevalence of limiting and disabling conditions is likely to be higher among the population in institutional care, prevalence estimates based solely on the non-institutionalised popu- lation are likely to underestimate prevalence for the full population, particularly at older ages. In the context of health expectancy calculations, one response to this situation has been to define institutional care as a distinct health state and to produce estimates of the expectation of life in institutional care, not in institutional care but with functional limitations or disability and not in institutional care and free of limitations or disabilities $[2,6]$. Surveys of the non-institutionalised population produce estimates of the conditional prevalence, Pr(limitation|non-institutionalised), which is exactly what is required to compute the expectation of life with non-institutionalised disability in a health expectancy calculation, partitioned as just discussed.

The strategy of defining institutional care as a distinct health state yields a coherent set of point-in-time health 
state prevalences and health expectancy estimates, covering the entire population. However changes in the prevalence of institutionalisation complicate the interpretation of changes in non-institutionalised health state prevalence. For example, in the context of declining utilisation of institutional care, it is likely that some individuals with limitations who would formerly have been cared for in institutions, now continue to live in the community, accessing disability support services as required. Thus, it is possible that any observed increase in non-institutionalised disability prevalence could be due to reductions in utilisation of institutional care rather than to increases in the underlying population prevalence. This issue is likely to be particularly relevant to the interpretation of changes in the prevalence of less severe limitations, because as access to institutional care becomes more limited it is people with less severe problems who are more likely to remain living in the community.

One approach to investigating the contribution of changes in institutionalisation prevalence to changes in non-institutionalised health state prevalence measures is to use sensitivity analysis. For example, by assuming that all individuals now living in the community who would formerly have been resident in institutions have some level of limitation, the maximum possible contribution of declining institutional care utilisation can be assessed. In this paper I use elementary conditional probability arguments to outline a method to compute adjusted prevalence estimates under such a scenario. Comparing adjusted and observed prevalences of non-institutionalised disability or limitation provides a means of assessing the likely impact of changes in the utilisation of institutional care on observed changes in non-institutionalised limitation prevalences. In order to simplify the subsequent presentation I focus on the case of declining utilisation of institutional care. While this is a plausible scenario for many health systems in the developed world, it may not be applicable in developing countries. However, arguments directly analogous to those outlined below yield an adjustment strategy to facilitate sensitivity analysis in the case of increasing utilisation of institutional care.

\section{Constructing adjusted health state prevalences for the case of declining utilisation of institutional care} Preliminaries

I assume health state prevalences for the non-institutional population are available for two time-points, together with information on the prevalence of institutional care for the same time points. The latter information will often be available from population census data, as is the case in New Zealand [6] and the United States [2]. For simplicity, I henceforth refer only to functional limitations, or to limitations, although the method clearly extends to disability or to any other health related state.
Let pins ${ }_{1}$ and pins 2 denote the prevalence of institutionalisation at time one and time two, respectively. Similarly let plim $_{1}$ and plim $_{2}$ denote respectively the limitation prevalences for the non-institutionalised population at the two time points. In addition, introducing specific indicator variables for limitation and institutionalisation status simplifies the subsequent development. Thus for times $\mathrm{i}=(1,2)$ let $Y_{i}$ be an indicator variable with $Y_{i}=1$ indicating limitation and $Y_{i}=0$ indicating absence of limitation.

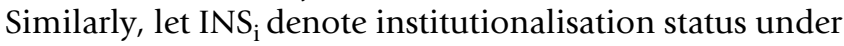
the conditions and policies operating at time $i$, with INS $_{\mathrm{i}}$ $=1$ indicating institutionalisation and $\mathrm{INS}_{\mathrm{i}}=0$ indicating residence in the non-institutionalised community.

Using this notation we have $\operatorname{plim}_{\mathrm{i}}=\operatorname{Pr}\left(Y_{i}=1 \mid \mathrm{INS}_{\mathrm{i}}=0\right)$ and pins $_{\mathrm{i}}$ is identified with $\operatorname{Pr}\left(\mathrm{INS}_{\mathrm{i}}=1\right)$, for $i=1,2$. Moreover, notation such as $\left(\mathrm{INS}_{1}=1, \mathrm{INS}_{2}=0\right)$ can be used to indicate subgroups of the population, in this case, the subpopulation living in the community at time two who would have been in institutional care under the conditions and policies operating at time 1 . Strictly speaking, the prevalences plim ${ }_{\mathrm{i}}$ and pins $\mathrm{s}_{\mathrm{i}}$ should be regarded as estimates of the probabilities $\operatorname{Pr}\left(Y_{i}=1 \mid \mathrm{INS}_{\mathrm{i}}=0\right)$ and $\operatorname{Pr}\left(\mathrm{INS}_{\mathrm{i}}\right.$ $=1$ ), however as this distinction has no practical implication for the subsequent discussion it is ignored. Uncertainty assessment is discussed briefly in the Discussion section, below

Our aim is to compute an adjusted limitation prevalence for time two, plim ${ }_{2}^{\text {adj }}$ say, which represents the expected prevalence for the non-institutionalised population at time two, under a scenario in which the underlying population prevalence of functional limitation does not change, but changes in the utilisation of institutional care change the composition of the non-institutionalised population. By comparing $\operatorname{plim}_{2}^{\text {adj }}$ with the observed prevalence plim $_{2}$, the impact of changes in institutional care utilisation on prevalence estimates for the non-institutionalised population can be assessed. Thus, our strategy is to compare observed prevalences at time two with those which would have been observed under a scenario in which utilisation of institutional care, but not population prevalence of limitation, changes between time one and time two.

\section{Derivation of the adjustment formula}

The non-institutional population at time two can be viewed as comprising two sub-populations defined in terms of residential status (institutional or community dwelling) under a counterfactual scenario in which all policies relating to access to institutional care at time one, remain unchanged through to time two. The first subpopulation comprises those individuals who would have been resident in the non-institutional community had the 
admission policies of time one been operating at time two, while the second sub-population comprises those individuals who would have been in institutional care had the policies of time one continued through to time two. As a shorthand, the latter group is henceforth referred to as the "de-institutionalised," population or the group "transferred" from institutional care to the non-institutionalised community, even though no physical transfer may have occurred. Similarly, where it simplifies the presentation, I refer to the sub-group of institutional care residents at time two, who would have been resident in the non-institutionalised community at time one as the group "transferred" from the community to institutional care.

Because they are defined in terms of a counterfactual scenario, the two sub-populations of the non-institutional population at time two are not identifiable via empirical observation. Nevertheless, conceptualisation of the noninstitutional population in terms of the sub-populations defined above is possible and proves useful in constructing adjusted prevalences

For the purposes of sensitivity analysis, we assume that a proportion, $\theta$, of the "de-institutionalised" population have some level of functional limitation, whereas the prevalence of functional limitation for the remainder of the community dwelling population at time two is identical to the corresponding prevalence at time one. These two assumptions can be written as follows:

$\operatorname{Pr}\left(\mathrm{Y}_{2}=1 \mid \mathrm{INS}_{2}=0, \mathrm{INS}_{1}=1\right)=\theta$

and

$\operatorname{Pr}\left(\mathrm{Y}_{2}=1 \mid \mathrm{INS}_{2}=0, \mathrm{INS}_{1}=0\right)=\operatorname{Pr}\left(\mathrm{Y}_{1}=1 \mid \mathrm{INS}_{1}=0\right)=\operatorname{plim}_{1}$ (2)

Setting $\theta$ in equation (1) equal to one, gives maximum weight to the proposition that observed increases in the prevalence of non-institutionalised limitation are attributable to declining utilisation of institutional care, by assuming that all "de-institutionalised" individuals have some level of limitation.

Now, given the institutionalisation probabilities, pins and pins ${ }_{2}$, only one constraint on the association between institutionalisation at time 1 and time 2 is required in order to fully specify the $2 \times 2$ table cross-classifying institutionalisation status under the conditions operating at the two time points. For example, with given marginal probabilities pins $_{1}$, and pins $s_{2}$, specification of a relative risk measure such as $\operatorname{Pr}\left(\mathrm{INS}_{2}=1 \mid \mathrm{INS}_{1}=1\right) / \operatorname{Pr}\left(\left(\mathrm{INS}_{2}=\right.\right.$ $1 \mid \mathrm{INS}_{1}=0$ ) or specification of an odds ratio for the association between institutionalisation status at the two time-points completely determines the four cells of the 2 $\times 2$ cross-classification [7]. Similarly, with given marginal probabilities, specification of any cell of the $2 \times 2$ classification determines the remaining cells. This situation is illustrated in table 1 , where the cell probability $\alpha$, can either be viewed as specified directly or derived from some other constraint on the association between institutionalisation status under the conditions operating at the two time points.

Table I: Cross-classification of institutionalisation risks under the policies operating at two time points.

\begin{tabular}{llll}
\hline & $\begin{array}{l}\text { Institutionalised } \\
- \text { time 2 }\end{array}$ & $\begin{array}{l}\text { Not-institutionalised } \\
- \text { time 2 }\end{array}$ & \\
\hline $\begin{array}{l}\text { Institutionalised - } \\
\text { time I }\end{array}$ & pins $_{2}-\alpha$ & pins $_{1}-$ pins $_{2}+\alpha$ & pins $_{\text {N }}$ \\
$\begin{array}{l}\text { Not } \\
\text { institutionalised - } \\
\text { time I }\end{array}$ & $\alpha$ & I-pins $-\alpha$ & I-pins \\
& & & \\
\cline { 2 - 4 } & pins $_{2}$ & I-pins & I \\
\hline
\end{tabular}

Clearly the parameter $\alpha$ in table 1 denotes the joint probability of institutionalisation under the policies operating at time two and non-institutionalisation (i.e. community dwelling) under the policies operating at time 1. Alternatively, $\alpha /\left(1-\right.$ pins $\left._{1}\right)$ represents the conditional probability of institutionalisation at time two given non-institutionalisation under the policies of time one, while $\alpha /\left(\right.$ pins $\left._{2}\right)$ represents the conditional probability of community dwelling under the conditions of time one, given institutionalisation under the conditions operating at time two.

Under a scenario of declining use of institutional care, the threshold for accessing institutional care presumably increases over time. Consequently, the probability that an individual offered institutional care at time two would have been assessed as able to live in the community under admissions policies operating at time one is low. Therefore, for the purposes of sensitivity analysis, it may not be unreasonable to set $\alpha$ to zero or to some value close to zero.

Now, applying straightforward conditional probability arguments, the prevalence of limitation at time two, for the community dwelling population at time two can be obtained as follows:

$\operatorname{Pr}\left(Y_{2}=1 \mid \mathrm{Ins}_{2}=0\right)=\left\{\begin{array}{l}\operatorname{Pr}\left(\mathrm{Y}_{2}=1 \mid \mathrm{INS}_{2}=0, \mathrm{INS}_{1}=0\right) \times P\left(\mathrm{INS}_{1}=0 \mid \mathrm{INS}_{2}=0\right)+ \\ \operatorname{Pr}\left(\mathrm{Y}_{2}=1 \mid \mathrm{INS}_{2}=0, \mathrm{INS}_{1}=1\right) \times P\left(\mathrm{INS}_{1}=1 \mid \mathrm{INS}_{2}=0\right)\end{array}\right\}$ 
Under the assumptions of equations (1) and (2) and Table 1, the right hand side of equation (3) is given by:

$\operatorname{plim}_{2}^{\text {adj }}=\operatorname{plim}_{1} \times \frac{\left(1-\text { pins }_{1}-\alpha\right)}{\left(1-\text { pins }_{2}\right)}+\theta \times \frac{\left(\text { pins }_{1}-\text { pins }_{2}+\alpha\right)}{\left(1-\text { pins }_{2}\right)}$

which involves only quantities assumed known and hence provides the requisite formula for computing functional limitation prevalences for time two, under the assumption of no change in limitation prevalence, except for "transfer" of individuals from institutional care to the non-institutionalised community.

The structure of equation (4) is instructive: The right hand side is a weighted average of the assumed limitation prevalence for the group who would be community dwelling under the conditions of both time one and time two (plim ${ }_{1}$, see equation (2)) and the limitation prevalence for the "de-institutionalised group" ( $\theta$, see equation one). The weights represent the proportions of the two groups in the community dwelling population at time 2. (Recall that we are assuming declining institutional care utilisation so that $\operatorname{pins}_{2}<$ pins $_{1}$ ). For any fixed value of $\alpha$, the adjusted prevalence increases with $\theta$ because $\theta$ represents the limitation prevalence for the "de-institutionalised" group and the greater the limitation prevalence for this group the greater the prevalence for the community dwelling population at time two.

For $\theta>\operatorname{plim}_{1}$, the adjusted prevalence increases with $\alpha$. This is because as $\alpha$ increases away from zero the probability of individuals "transferring" from the communitydwelling to the institutionalised populations increases, and in order to meet the requirement of an overall decline in institutional care utilisation, a correspondingly greater number of individuals must transfer from institutional care to community dwelling, bringing with them their assumed higher prevalence of limitations $\left(\theta>\operatorname{plim}_{1}\right)$. In the unlikely event that $\theta$ is less than plim $_{1}$ the adjusted prevalence would decline with increasing $\alpha$ because individuals "transferring" into the non-institutional population would have lower limitation prevalence than those "transferring" out.

In practice, age-specific adjusted prevalences would often be required in which case equation (3) should be interpreted as referring to the adjustment formula for a specific age group.

\section{Examples}

\section{Example I}

I consider first an example based on New Zealand institutionalisation and limitation prevalence data for the years 1981 and 1996. Graham et al [6] report that the prevalence of moderate agility limitation among females aged 65 or over increased from $13 \%$ to $17.2 \%$, while the utili- sation of institutional care declined from $8.3 \%$ to $7.5 \%$. Intuitively, such a small decline in institutional care utilisation seems unlikely to account for the observed increase in the prevalence of agility limitation.

Nevertheless, it is useful to quantify the possible contribution of declining institutionalisation to the observed increase in limitation prevalence. If we assume that all "de-institutionalised" individuals have some level of agility limitation and that no individuals in institutional care in 1996 would have been community dwelling under 1981 conditions, then $\theta=1$, and $\alpha=0$. From equation (3) the adjusted prevalence for 1996 is $13.8 \%$, only marginally greater than the actual prevalence for 1981 . Thus even assuming that all "de-institutionalised" individuals had some level of limitation it seems unlikely that the observed increases in the prevalence of non-institutionalised limitation can be explained by people who would formerly have been in institutional care living in the noninstitutionalised community in 1996.

Allowing for the possibility that some "de-institutionalised" individuals may be free of agility limitation $(\theta<1)$ brings the adjusted prevalence even closer to the 1981 figure. For example setting $\theta=0.9$ yields an adjusted prevalence of $13.7 \%$ and setting $\theta=0.5$ yields an adjusted prevalence of $13.3 \%$

It appears that declining utilisation of institutional care could explain the increase in non-institutionalised limitation prevalence only under the unrealistic scenario in which there is an appreciable probability that individuals in institutional care in 1996 would have been living in the community under the conditions operating in 1981. For example with $\theta=1$, setting $\alpha=0.5 \times$ pins $_{2}$ so that $\operatorname{Pr}\left(\mathrm{INS}_{1}\right.$ $\left.=0 \mid \mathrm{INS}_{2}=1\right)=0.5$, yields an adjusted prevalence of $17.3 \%$.

The numerical results discussed above have clarified that, under plausible assumptions concerning the decline in institutional care utilisation, the decline cannot account for the increase in limitation prevalence, and could only do so under implausible assumptions.

\section{Example 2}

As a second example I consider a situation similar to that described in example one, except that a more marked decline in utilisation of institutional care is assumed by setting pins ${ }_{2}=4.2 \%$. Considering initially the case with $\theta$ $=1$, and $\alpha=0$, the adjusted prevalence computed via equation (3) is $16.7 \%$, close to the observed figure of $17.2 \%$ for 1996 . Thus, in this, hypothetical, case it seems possible that a substantial proportion of the observed increase in prevalence could be attributed to declining institutional care prevalence, under the assumption that a 
high proportion of "de-institutionalised" individuals have an agility limitation. However, in order for the adjusted prevalence to equal or exceed the observed prevalence for 1996 the probability for individuals in institutional care in 1996 living in the community under 1981 conditions has to be appreciable. For example, setting $\alpha=$ $0.25^{*}$ pins $_{2}$, so $\operatorname{Pr}($ INS1 $=0 \mid$ INS2 $=1)=0.25$, yields adjusted prevalences of $17.7 \%$ for $\theta=1,17.1 \%$ for $\theta=0.9$ and $15.0 \%$ for $\theta=0.5$. Overall, declining use of institutional care is a more plausible explanation for the observed increase in non-institutionalised limitation prevalence in example two than in example one.

\section{Discussion}

This paper has outlined a method for constructing adjusted non-institutionalised health state prevalences, under the assumption of no change in prevalence, except for the impact of de-institutionalisation on the composition of the non-institutional population. These adjusted prevalences facilitate sensitivity analysis to assess the impact of declining institutionalisation on changes in the prevalence of non-institutionalised health states. Although not emphasised here, the adjusted prevalences can be used as input to sensitivity analyses exploring the impact of changing utilisation of institutional care on summary measures of population health which combine information on health state prevalence and mortality. Graham et al, [6] provide an illustration of this in the case of health-state expectancies. Sensitivity analyses for summary measures employing severity or preference weighting of health states, such as health adjusted life expectancy (HALE) [8] are also possible, provided plausible average weights for the institutional population are available for the time periods being compared.

While the focus of this paper has been on the impact of temporal changes in the utilisation of institutional care, similar issues to those discussed above arise in crossnational comparisons or analyses of social variation in disability or functional limitation prevalence. If the utilisation of institutional care varies between countries, or between groups within a country, then comparisons of limitation prevalence based on surveys of the non-institutionalised population are potentially misleading. Sensitivity analysis based on equation (3) could be used to explore the possibility that observed differences in the prevalence of non-institutionalised limitation could be attributable to differential access to institutional care.

When the prevalences of institutionalisation and noninstitutionalised health states are unknown but estimates are available, the adjustment formula of equation (3) can be applied using the relevant estimates in place of pins ${ }_{i}$ and $\operatorname{plim}_{\mathrm{i}}, i=1,2$. Moreover, the adjustment formula can be embedded in whatever procedure is adopted to account for uncertainty in these estimates.

In particular, under a Bayesian approach to inference, implemented via Monte Carlo simulation, (for example see Salomon et al [9]) equation (3) can be applied to each draw from the posterior distribution of the limitation and institutionalisation prevalences to generate a joint posterior distribution for the observed and adjusted prevalences. Based on this posterior distribution, statistics such as the posterior probability that observed prevalence exceeds that computed under a given sensitivity analysis scenario can be computed [6]. This provides a convenient summary of the evidence for observed prevalence exceeding that predicted under a given sensitivity analysis scenario. A further advantage of adopting a Bayesian approach to inference is that when a single inference is desired rather than a series of "what-if" style sensitivity analyses, uncertainty concerning the unknown sensitivity parameters, $\alpha$ and $\theta$, can be accommodated. By constructing plausible prior distributions for the sensitivity parameters and incorporating these priors into a Monte Carlo posterior approximation algorithm, a posterior distribution reflecting uncertainty concerning both the sensitivity parameters and the institutionalisation and limitation prevalences, can be obtained.

\section{Conclusion}

The adjustment formula presented in equation (4) above facilitates the use of sensitivity analysis to explore the potential impact of declining utilisation of institutional care on changes in non-institutionalised health state prevalence.

\section{Competing interests}

None declared.

\section{Authors' contributions}

PG was solely responsible for this work.

\section{Acknowledgements}

This study was funded by the Health Research Council of New Zealand.

\section{References}

I. Sullivan DF: A single index of mortality and morbidity. HSMHA Health Rep 1971, 86:347-354.

2. Crimmins EM, Saito $Y$ : Trends in healthy life expectancy in the United States, 1970-1990: gender, racial and educational differences. Social Science \& Medicine 200I, 52:1629-164I.

3. Cambois E, Robine JM, Hayward MD: Social inequalities in disability-free life expectancy in the French make population, 1980-I99 I. Demography 200I, 38:5I3-524.

4. Sihvonen Ari-Pekka, Kunst AE, Lahelma E, Valkonen T, Mackenbach JP: Socioeconomic inequalities in health expectancy in Finland and Norway in the late 1980s. Social Science \& Medicine 1998, 47:303-315.

5. Davis P, Graham P, Pearce N: Health expectancy in New Zealand, $1981-1991$ : social variations and trends in a period of rapid social and economic change. J Epidemiol Community Health 1999, 53:519-527. 
6. Graham P, Blakely T, Davis P, Sporle A, Pearce N: Compression, expansion or dynamic equilibrium? - The evolution of health expectancy in New Zealand. Journal of Epidemiology \& Community Health 2004, In Press:

7. Lee A: Generating random binary deviates having fixed marginal distributions and specified degrees of association. American Statistician 1993, 47:209-2I5.

8. Mathers CD: Health Expectancies: An overview and critical appraisal. Summary Measures of Population Health: Concepts, Measurement, Ethics and Applications Edited by: Murray C J L, Salomon J A, Mathers CD and Lopez A D. Geneva, WHO; 2002:178-203.

9. Salomon JA, Mathers CD, Murray CJ, Ferguson B: Methods for life expectancy and healthy life expectancy uncertainty analysis. Global Programme on Evidence for Health Policy Working Paper No. 10. Geneva, World Health Organization; 200I.

Publish with Bio Med Central and every scientist can read your work free of charge

"BioMed Central will be the most significant development for disseminating the results of biomedical research in our lifetime. "

Sir Paul Nurse, Cancer Research UK

Your research papers will be:

- available free of charge to the entire biomedical community

- peer reviewed and published immediately upon acceptance

- cited in PubMed and archived on PubMed Central

- yours - you keep the copyright

Submit your manuscript here:

http://www.biomedcentral.com/info/publishing_adv.asp 